Bedeutung von Selbstbestimmung aus christlicher Sicht im Spannungsfeld zwischen Individuum und Gesellschaft $^{1}$

\section{Paul Kleiner}

Das Thema unserer Fachtagung benennt den Gegenstand unserer Beschäftigung: Selbstbestimmung - mit dem Fremdwort «Autonomie». Und zwar das Spannungsfeld Selbstbestimmung, was hoffentlich heute zu interessanten Diskussionen und hilfreichen Gedankenanstössen führen wird. Ein spezifisches Spannungsfeld ist die Frage nach dem Fördern und Fordern: Wer fördert Selbstbestimmung bei wem? Der Einzelne bei sich selber? Die Fachperson der Sozialen Arbeit bei der Klientel? Die Institution der Sozialen Arbeit oder gar der Staat bei allen Beteiligten und Betroffenen? Fördert der andere, ob nun Fachperson oder Mit-Klientel oder sonstige Menschen aus dem persönlichen Bezugssystem, meine Selbstbestimmung? Oder unsere? Oder wird die Selbstbestimmung dieser verschiedenen Personen im Miteinander gegenseitig gefördert? Usw. Dasselbe könnte ich beim Fordern durchspielen: Wer fordert Selbstbestimmung von wem?

Das Thema begrenzt gleichzeitig: Selbstbestimmung in der christlichen Sozialen Arbeit, also nicht in der Schweizer Politik (Stichwort: fremde Richter) oder im Schulwesen (Stichwort: autonome Schule) oder sonst irgendwo.

Das Thema spitzt zu: In der christlichen Sozialen Arbeit, also nicht nur allgemein in der Sozialen Arbeit, sondern spezifisch und explizit in der christlichen. Was «christliche Soziale Arbeit» bedeutet, ist nun wiederum

1 Der folgende Aufsatz wurde in abgekürzter Fassung an der Fachtagung «Spannungsfeld Selbstbestimmung in der christlichen Sozialen Arbeit» vom 23.3.2018 vorgetragen. Der mündliche Vortragsstil ist weitgehend beibehalten. nicht explizites Thema von heute, sondern der implizite Ansatz des Organisationskomitees, der hoffentlich durch die gemeinsame Arbeit an den vergangenen Fach- sowie Arbeitstagungen und auch heute weiter Konturen gewinnt.

In einem ersten Teil wende ich mich dem Selbst im Spannungsfeld von Individuum und Gesellschaft zu: Was ist das überhaupt? Also grundlegende anthropologische Überlegungen. Im zweiten Teil geht es ethisch weiter: Selbstbestimmung als ein guter, gelingender Umgang mit dem eigenen und mit andern «Selbst».

\section{1. «Selbst» - was ist das? (Anthropologie)}

Beginnen wir mit zwei unterschiedlichen Definitionen. Das Dorsch Lexikon für Psychologie versteht unter Autonomie die Fähigkeiten einer Person, ihr «Leben unabhängig von andern zu gestalten, indem sie selbständig Ziele setzt, und ihr Erleben und Verbalten eigenverantwortlich unter Berücksichtigung eigener Werthaltungen (Werte) reguliert.»² Gerrig wiederum referiert in seinem dicken Lehrbuch Psychologie ${ }^{3}$ das Selbstkonzept von William James, wozu das soziale Ich gehört als Bewusstsein, wie andere mich sehen; ebenfalls bringt er ein Unterkapitel über die kulturelle Konstruktion des Selbst. Bei Gerrig ist das Selbst des Individuums eingebettet in das Grössere von Mensch-Umwelt-System (sozial/kulturell), Kracke hingegen fokussiert beim Selbst ganz stark das Individuum in seiner Selbstbestimmung bzw. (selbstverständlich positiven!) Autonomieentwicklung: «unabhängig von andern ... selbständig ... eigenverantwortlich ... eigene $[\mathrm{r}]$ Werthaltungen».

2 Bärbel Kracke, Autonomieentwicklung, in: Markus Antonius Wirtz (Hg.), Dorsch Lexikon der Psychologie, Bern 162013, 229-230 (229).

3 Richard J. Gerrig/Philip G. Zimbardo, Psychologie, Hallbergmoss 182008, 531-537. 
1.1 Das menscbliche «Selbst» als Individuum und soriales Wesen

Die beiden Definitionen oder Konzepte oder Konstruktionen von Selbst müssen sich nicht zwingend widersprechen, legen das Augenmerk aber auf zwei unterschiedliche Pole: Das Selbst als Kern des einzelnen Menschen bzw. das Selbst als Produkt der Interaktion des einzelnen Menschen mit seiner materiellen, sozialen und kulturellen Umwelt. In diesem Spannungsfeld erfahren sich Menschen und wird das Selbst verortet:

Der Mensch als Individuum, als einzigartig, unterschieden von den andern, eben er bzw. sie selber, einmalig, das unteilbare (in-dividuum) und unhintergehbare Grundelement der Menschheit und Gesellschaft und Generationenfolge und Familienverbände. Ich selbst! Selb-ständig: Als Selbst stehe ich da.

Der Mensch als soziales Wesen, eingebunden in Beziehungen, in stetem Geben und Nehmen, interdependent, angewiesen auf andere und gleichzeitig einwirkend auf andere, bedürftig und mächtig. Wir selbst! Selbstwerdung: Als Selbst entwickle und entfalte ich mich im gegenüber zu anderen Selbst.

Der geistesgeschichtliche Aufstieg der Betonung des Individuums in der westlichen Welt ist verbunden mit Namen wie Descartes («ich denke, also bin ich») und Kant («Habe Mut, dich deines eigenen Verstandes zu bedienen»). Das Konzept des Menschen als Individuum, die Betonung dieses einen Pols, verstand sich als eine emanzipatorische Bewegung. Eine geistige und politische Befreiung des Individuums von absolutistisch-klerikal-feudalen Bindungen und Beschränkungen. Ein solches individuelles Selbst kann, darf und soll sich selbst bestimmen, gemäss seinem eigenen Willen, Verstand und Wertmassstäben leben. Das individuelle Selbst wird zum Kern des Menschen - manchmal verbunden mit Begriffen wie «natürlich» oder «angeboren» (bei der allgemeinen Erklärung der Menschenrechte), was dann an ein vorgesellschaftlich gegebenes Einzelwesen denken lässt.

Letzteres aber ist eine Abstraktion von realen Erfahrungen, wie der andere gedankliche Pol zum Selbst sofort zur Geltung bringt. Philoso- phen wie Martin Buber («Ich werde am Du») oder Emmanuel Lévinas («Die Spur des Anderen») bringen dies zur Sprache. Nicht einmal die einmalige genetische Codierung des Menschen ist ein biologisch fester Kern des Individuums; vielmehr entwickelt sich der Mensch schon biologisch im Zusammenspiel von Genen und Umwelt. Jede und jeder Einzelne wird als Baby in die menschliche Gemeinschaft hinein geboren, wird sozialisiert und erlernt die Muttersprache und Kultur von anderen Menschen. Ich bzw. Selbst sind ohne Anderes und Andere mit einer gemeinsamen Geschichte nicht zu haben. ${ }^{4}$

Jetzt kann man vom einen Pol zum andern kippen und versuchen, gedanklich - philosophisch - weltanschaulich aus der Ellipse sozusagen einen Kreis zu machen. Zum Beispiel so:

Westlicher liberaler Individualismus: Das individuelle Selbst ist zuerst und unabhängig und gegeben: Mit Würde, mit Rechten, mit Interessen. Die Selbst-bestimmung und -entfaltung ist das höchste Lebensziel. Das Umfeld bzw. die Umwelt des Selbst (materiell, sozial) soll dem Selbst dienen und es möglichst nicht einengen. Die einzig legitime Begrenzung des Selbst ist das Lebensrecht anderer Individuen.

Antikes Griechentum: Der Kosmos ist zuerst und unabhängig und gegeben. Das individuelle Selbst repräsentiert als Mikrokosmos den Makrokosmos. Das höchste Lebensziel ist, sich in die «natürliche» kosmische Ordnung einzufügen. Dieses ermöglicht erst ein wahres Selbst, das in Harmonie mit sich und allen andern lebt. Oder Kollektiv-Kulturen und -Ideologien: Vor 80 Jahren hiess es in Deutschland «du bist nichts, dein

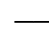

4 Vgl. auch Alasdair C. MacIntyre, After Virtue, Notre Dame 1981; Edi Martin (Selbstbestimmung, das ist gut! Was genau ist daran und was hat Selbstbestimmung mit Sozialer Arbeit zu tun? Referat vom 27. November 2008 bei AvenirSocial Zürich, URL: http://www.avenirsocial.ch/cm_data/vortrag-martin.27.11.08.pdf, abgerufen am 24.1.2017, 6) formuliert so: «Menschliche Individuen sind partiell autonom und partiell sozial abhängig» 
Volk ist alles». Das Selbst fand seine Bestimmung darin, dem Volk als Grösseren zu dienen. Diese umfassendere Grösse für das Individuum kann auch die Familie/Clan, die Nation, die religiöse Gruppe usw. sein.

Anders gesagt: Die Erfahrung von Selbst und Anderen dreht sich im Kreis.

Einmal ist der/die Andere einengend und bedrohend für mein Selbst. Die Mutter steckt das Kind in ein Laufgitter. Der Staat mit seiner Bürokratie macht mich zu einer vielstelligen Nummer, die Steuern zahlt, sich versichert, den Wohnort meldet. Die Arbeitsstelle fordert acht Stunden täglich plus ganz viel Lebensenergie. Die Partnerin erwartet Zuwendung und Verständnis. Und früher schrieb die Kirche die Dogmen vor, exkommunizierte oder verbrannte Freigeister. Darum ein Hoch auf das Individuum, das sich seine Freiheit und Selbständigkeit immer wieder erkämpfen muss.

Dann ist der/die Andere bereichernd für mein Selbst, ermöglicht es und lässt es aufblühen. Die Mutter nährt und liebt das Baby. Der Staat sorgt für äussere Sicherheit und Stabilität, stellt ein Bildungs- und Gesundheitssystem zur Verfügung. Die Arbeitsstelle bietet Raum zum Wirken, Gestalten, Erschaffen und für vielfältige Begegnungen. Der Partner gibt Zuwendung und Verständnis. Die Kirche vermittelt Trost und Orientierung in stützender Weggemeinschaft. Darum ein Hoch auf die Sozialität des Menschseins, welche dem Individuum Gemeinschaft und Halt gibt sowie ein Handlungsfeld für Selbständigkeit eröffnet.

Beides ist wahr: Andere bedrohen mein Selbst und ermöglichen es. Besser eine Ellipse als ein Kreis. Oder umgekehrt formuliert: Das Selbst als Person existiert in Selbstbesitz und Selbstdistanz: Selbstbesitz als Freiheit von Mächten oder sozialen Gruppen; die Person gehört sich selbst. Und Selbstdistanz, weil die Person über sich selbst verfügt und «erwacht in Begegnung mit anderer Selbstgehörigkeit».5 Das Selbst als Person ist dynamisch: Selbst-sein (Souveränität) und Selbsttranszendieren (Hingabe, sich mit-teilen) bzw. Bewährung am Andern. Das Selbst als Person verharrt nicht nur im Subjekt-Objekt-Schema mit einer (den/das Andere) abwehrenden Selbstbewahrung, sondern «es beginnt ein Sich-Aussetzen und Ausgesetztsein: Person wird auf Person resonant.» ${ }^{6}$

\subsection{Biblisch-theologische Überlegungen}

Biblisch-theologische Überlegungen lassen sich auch in die bisher beschriebene Polarität einzeichnen: Das Selbst mit den beiden Brennpunkten autonomes Individuum und Interaktion/Gemeinschaft. Ausdrücklich bringt die christliche Reflexion Gott als grundlegenden Player für das menschliche Selbst ins Spiel. Aus menschlicher Sicht ist Gott ein weiteres Element des Umfelds bzw. Systems und entsprechend anschlussfähig für den säkularen wissenschaftlichen Diskurs, in dem sich das Selbst bewegt. Aus theologischer Sicht hingegen ist der dreieine Gott der Bibel Schöpfer von Mensch und Welt, Ursprung der menschlichen Gottebenbildlichkeit und des Person-Seins, bleibendes Gegenüber in seiner Selbstoffenbarung für den Menschen als Individuum und soziales Wesen und damit nicht ein Element unter anderen bzw. (im Bild eines Spiels zu sprechen) nicht ein weiterer Mitspieler oder Gegnerin auf dem Spielfeld, sondern Spielertrainer, Besitzerin des Stadions und Erfinder des Spiels in einem.

Im Folgenden nehme ich eine theologische Perspektive ein, versuche aber zwischendurch, den Blickwinkel zu wechseln bzw. in die sozialwis-

5 Hanna-Barbara Gerl-Falkowitz, Frau - Männin - Menschin. Zwischen Feminismus und Gender, Kevelaer 2009, 196.

6 Ebd., 197. 
senschaftliche Perspektive zu übersetzen. Biblisch-theologische Überlegungen können grundsätzlich in vier Akte eines Dramas gegliedert werden, so auch zum Selbst des Menschen:

1.2.1 Schöpfung: Der Mensch ist geschaffen zu Gottes Ebenbild Die Bibel versteht den Menschen als Geschöpf. Jede und jeder Einzelne ist individuell von Gott geschaffen (Ps 139,13) und sein/ihr Leben soll nicht von andern angetastet werden bzw. wird von Gott als wertvol geschützt (1. Mose 9,6). Zudem ist das Individuum als Leib-SeeleEinheit geschaffen (1. Mose 2,7) und ein mit sich selbst identisches Selbst durch die Geschichte (von der Wiege bis zur Bahre - und kraft der Auferweckung durch Gott sogar über den Tod hinaus). Diese Kontinuität und Identität des Selbst durch die Zeit hindurch wird beispielsweise deutlich an der Verantwortung des Individuums vor Gott (Dan 12,13; Mt 12,36; 1. Kor 15,58).

Der Mensch als Geschöpf ist grundsätzlich abhängig von Gott, der es erschaffen hat und erhält, ${ }^{7}$ und eingefügt in interdependente Beziehungen mit Mitmenschen und der Mit-Schöpfung (vgl. 1. Mose 2: Der Mensch im Garten als Mann und Frau). Das Selbst ist ein Beziehungswesen, eine Person geschaffen im Ebenbild Gottes, der als Geheimnis der Dreieinigkeit sich selbst ist und sich selbst transzendiert in Hingabe zum andern.

Die Menschen, gemäss biblischem Zeugnis und durchaus sozialwissenschaftlich anschlussfähig, sind also gleichzeitig Individuen und Beziehungswesen. Sie sind gleichzeitig unendlich wertvoll und begrenzt, bedürftig sowie beauftragt zu handeln.

$\mathrm{Zu}$ dieser Grund-Polarität kommt eine zusätzliche zeitliche Dynamik hinzu: Die Menschen sind als Geschöpfe geschaffen (Anfang) mit einer

7 In der Theologie wird das Erhaltungshandeln als «creatio continua» bezeichnet, vgl Ps 104.
Bestimmung (Ziel). In gesamtbiblischer Perspektive könnte ich formulieren: Gott möchte die Menschen - zusammen mit der ganzen Schöpfung - in seinem Reich dabei haben. ${ }^{8}$ Oder mit den Worten eines klassischen reformierten Bekenntnistextes, dem grossen Westminster Katechismus von 1647: «Die vornehmste und höchste Bestimmung des Menschen ist, Gott $\mathrm{zu}$ verherrlichen und ihn vollkommen zu geniessen in alle Ewigkeit.»9

Zusammengefasst: Die Polarität des Menschen als Individuum und Beziehungswesen entfaltet sich dynamisch in einer Geschichte vom Jetzt zur zukünftigen Bestimmung und spielt sich ab im Viereck wertvoll begrenzt - bedürftig - beauftragt.

\subsubsection{Sündenfall: Der Mensch will «sein wie Gott»}

In der Bibel ist der Gottesbezug das Wesentlichste für alles Geschaffene, auch für den Menschen. Dies ist ein Spezifikum der theologischen Rede vom Menschen. Dieser Gottesbezug ist nun nicht hinreichend erfasst mit dem Begriff des Geschöpfs, wie eben beschrieben. Gemäss der Bibel hat der Mensch in Ungehorsam gegen Gott rebelliert (1. Mose 3,6-7) und ihn vertauscht mit der Schöpfung (Römer 1,23); Gott hat ihn dann aus dem Paradies vertrieben (1. Mose 3,23f.) bzw. ihn sich selbst überlassen (Römer 1,24.26.28: «dahingegeben»). Diesen Aspekt des Menschseins nennt die Bibel Sünde, die das ganze Menschsein (1. Mose 8.22 «von Jugend auf», Ps 51,7 «geboren» und «empfangen») und alle Menschen (Röm 3,9.19f.) umfasst.

8 Vgl. Karl Barth: Die Schöpfung zielt auf den Bund (Kirchliche Dogmatik III/1, §41).

9 URL: http://files.feedplace.de/kvetterli/Grosser\%20Westminster\%20Katechismus. pdf, aufgerufen am 7.11.2016. Im kleinen Westminster Katechismus lautet das so: «Das höchste Ziel des Menschen ist, Gott zu verherrlichen und sich für immer an ihm zu erfreuen.» (URL: http://www.bucer.org/uploads/tx_org/mbstexte061.pdf, abgerufen am 7.11.2016) 
Das von der Sünde gezeichnete Selbst des Menschen verfällt der Selbstentfremdung und Selbsttäuschung; es verfehlt die «geschöpfliche[n] Bestimmung [...] in der Sünde durch Selbstvergötzung und das Streben nach Selbsterlösung». ${ }^{10}$ Dies betrifft den Menschen als Individuum (Selbstbezug) und als Beziehungswesen (Selbst gegenüber andern). Bildlich gesagt: Die Ellipse wird immer wieder zum Kreis verformt.

1.2.3 Erlösung: In Jesus Christus versöhnt Gott die Welt (inklusive Menschen) mit sich selber

Die Bibel bleibt aber nicht bei der statischen Doppelaussage des Menschen als Geschöpf und Sünder. Gott ist nicht lediglich der Erhalter seiner Schöpfung angesichts der Sünde. Vielmehr wohnt der Bibel die Dynamik einer fortscheitenden Geschichte inne, deren Mitte Gottes Selbstoffenbarung in Jesus von Nazareth ist. Wie die Sünde den Menschen als Individuum (inklusive Selbst-bezug) und als (soziales) Beziehungswesen (zu Gott, zu Mitmenschen und zur Mitschöpfung) betrifft, so wirkt auch das Heil durch Jesus Christus in alle Dimensionen des Selbst: Frieden mit Gott (z.B. Röm 5,1), Heilung des Leibes und der «Seele» (z.B. Hoffnung in Röm 5,2; Trost in 2. Kor 1,3ff; gesunde Identität in 2. Tim 1,7), Frieden zwischen Menschen und Gruppen (z.B. Eph $2,14 \mathrm{ff})$.

Der Mensch erfährt diese Erlösung im Glauben real schon jetzt - das Reich Gottes ist angebrochen in Jesus Christus (Mk 1,15) - und gleichzeitig bedeutet Glauben noch nicht Vollendung. Noch herrscht der Kampf zwischen Geist und Fleisch (Röm 7) und ist der Mensch gleichzeitig gerecht und Sünder (Martin Luther: simul justus et peccator).

10 Kirsten Huxel, Art. Selbst. III. Dogmatisch; in: RGG 7 (42008), 1154f. (1154).
1.2.4 Vollendung: Der neue Himmel und die neue Erde

In der Vollendung ist der Mensch immer noch sich selber. Die Bibel bezeugt sehr deutlich die Kontinuität des Individuums durch Tod und Auferweckung hindurch. Zum Beispiel wird Daniel nach einem langen Leben getröstet: «Ruhe, bis du auferstehst zu deinem Erbteil am Ende der Tage». (12,13; vgl. 1. Kor 15,58: «Euer Werk ist nicht vergeblich in dem Herrn».) Oder Jesus verheisst dem Mann am Kreuz neben ihm: «Heute wirst du mit mir im Paradies sein.» (Lk 23,43)

Gleichzeitig wird die Vollendung vollkommen unvorstellbar sein Ohne Tod, Krankheit, Tränen und Leid (Offb 21,4), ohne Heiraten und Verheiratet-Werden (Mt 22,30). Die Verschiedenheit von Individuen und Kulturen bleiben bestehen (z.B. Offb 7,9), jedoch ohne Streit und Neid, sondern in reiner Bereicherung, Schönheit und Vielfalt. Das Pfingstwunder war eine Vorwegnahme der Vollendung: Die Pilger aus aller Welt hörten/verstanden die aramäisch sprechenden Apostel in Jerusalem in ihren eigenen Muttersprachen, die weit Hergereisten mussten nicht etwa alle das lokale Aramäisch, die Sprache von Jesus lernen! (Apg 2,6-11)

Ich fasse diese vier Akte einer dynamischen biblischen Anthropologie des Menschen als Geschöpf des dreieinen Gottes zusammen: ${ }^{11}$

1. Wir sind gewollt, als selbständige Individuen in Zugehörigkeit zu Andern: wertvoll - begrenzt - bedürftig - beauftragt.

2. Wir sind gefährdet, wie mit einer angeborenen Immunschwäche.

3. Wir sind geliebt, mit heilender und erlösender Kraft.

4. Wir werden erwartet, in der vollendeten Herrschaft Gottes von Friede und Gerechtigkeit.

Nun wende ich mich dem zweiten Teil mit ethischen Überlegungen zu.

11 In Anlehnung an Susanne Heine, Koordinaten des Daseins - Die christliche Matrix in: Frank Worbs (Hg.), «Ganz Mensch bis zum Tod», Zürich 2009, 11-28. 


\section{Selbstbestimmung - gelingendes Leben mit dem eigenen und anderen Selbst (Ethik)}

\subsection{Der Grundsatz der Selbstbestimmung}

Im gesellschaftlichen Mainstream und vermutlich auch in den meisten theoretischen Erwägungen der Sozialen Arbeit ist Autonomie bzw. Selbstbestimmung des Individuums und insbesondere der Klientel der Sozialen Arbeit ein selbstverständlicher und fundamentaler Wert. In der Einladung zu dieser Fachtagung wurde es so formuliert:

«Selbstbestimmung wurde in den letzten Jahrzehnten zu einem zentralen Wert in unserer Gesellschaft. Nach und nach wurden entsprechende Anliegen auch in der Sozialen Arbeit gefordert und konzeptionell umgesetzt. Die Ratifizierung der UN Behindertenrechtskonvention durch die Schweiz war in dieser Hinsicht ein weiterer Meilenstein.

[...] in den letzten Jahrzehnten [wurden] eine Reihe von Forderungen zur Selbstbestimmung mit einem (aus heutiger Sicht) grossen Konsens und auch in Abgrenzung $\mathrm{zu}$ früheren fremdbestimmten Interventionen übernommen (angefangen beim Einbezug der KlientInnen bei 〈grossen〉 Entscheidungen z.B. in der Wahl der Institution bis hin zu «kleinen〉 Entscheidungen im Alltag wie der Wahl der Kleidung)».

Ein Blick in den Berufskodex Soziale Arbeit Schweiz soll als Beleg genügen, dass Selbstbestimmung heute ein, wenn nicht sogar der Grundwert in westlichen Gesellschaften ist. Dort erscheint der «Grundsatz der Selbstbestimmung» ${ }^{12}$ unmittelbar nach dem «Grundsatz der Gleichbehandlung» ${ }^{13}$, am Anfang des Teils zu den Grundwerten der Sozialen Arbeit. Selbstbestimmung wird in der Tradition des westlichen liberalen

12 Avenir Social, Berufskodex Soziale Arbeit Schweiz, Bern 2010, URL http://www.avenirsocial.ch/cm_data/Do_Berufskodex_Web_D_gesch.pdf, 8 .

13 Ebd.
Individualismus definiert, also mit dem Fokus auf das Individuum und nicht auf den Menschen als soziales Wesen, was eigentlich im Kontex sozialer Arbeit erstaunlich ist: «Das Anrecht der Menschen, im Hinblick auf ihr Wohlbefinden, ihre eigene Wahl und Entscheidungen zu treffen, geniesst höchste Achtung, vorausgesetzt, dies gefährdet weder sie selbst noch die Rechte und legitimen Interessen Anderer.» ${ }^{14}$

Kontext oder Hintergrund dieser Betonung der Selbstbestimmung in der Sozialen Arbeit ist deren Bewusstsein, dass sie sich meistens in asymmetrischen Beziehungen mit einem Machtgefälle bewegt - und darum den emanzipatorischen Impuls von Selbstbestimmung gegenüber subtiler oder offener negativer Machtausübung, sei sie individuell oder strukturell, besonders hochhält.

\subsection{Dilemmata und Differenzierungen}

Nur: So einfach ist es nicht. Denn der Berufskodex ${ }^{15}$ zählt unmittelbar vorher verschiedene Dilemmata auf. Das Recht auf Selbstbestimmung kann in Spannung stehen zur momentanen oder dauerhaften Unfähigkeit, dieses Recht wahrzunehmen. Oder noch fundamentaler kann die Selbstbestimmung eines Menschen in Konflikt geraten mit der Überzeugung eines anderen Menschen, dass ersterer des Schutzes und der Fürsorge Sozialer Arbeit bedarf - und darum allenfalls eine Fremdbestimmung vertritt oder gar durchsetzt! ${ }^{16}$ Die Überlegungen verharren aber auch hier beim Individuum-Pol des Selbst und der Selbstbestimmung:

14 Ebd.

15 Avenir Suisse, Berufskodex (Anm. 12), 7

16 Vgl. Martin, Selbstbestimmung (Anm. 4), 10: «Für Professionelle der Sozialen Arbeit ist es nur dann legitim diese selbst-determinierten Regulationen zu stören, wenn eine Person durch ihr Verhalten und Handeln sich oder andere in der Befriedigung ihrer Bedürfnisse behindert. Dann sollen Professionelle begrenzende Macht einsetzen». 
Das Individuum kann unfähig zur Selbstbestimmung sein; in solchen Fällen darf (oder muss) Fremdbestimmung ins Spiel kommen.

Es finden sich aber auch weitere Stimmen im Diskurs der Sozialen Arbeit zur Selbstbestimmung, welche die Spannungsfelder noch weiter differenzieren und vertiefen. Der Sozialalmanach 2007 der Caritas widmete sich dem Konzept der Eigenverantwortung. ${ }^{17}$ In einem Beitrag reflektiert Walter Schmid, damals Präsident der Schweizerischen Konferenz für Sozialhilfe und Direktor der Hochschule Luzern Soziale Arbeit, über das Konzept des Empowerment: ${ }^{18}$ Es ist ein zentraler Orientierungspunkt für die Soziale Arbeit; Selbstbestimmung der Klientel ist das grundlegende Ziel Sozialer Arbeit. Nur: Obschon Empowerment die Machtbalance tangiert - weg von paternalistischer Hilfe hin zu Augenhöhe zwischen Professionellen und Klientel, mit gemeinsamem Dialog und Aushandlungsprozessen -, besteht erstens die Gefahr, dass die Rede von Empowerment gleichzeitig die Machtverhältnisse verschleiert; denn Soziale Arbeit hat auch ein Mandat «seitens der Gesellschaft und der

17 Mehrheitlich kritisch wird Eigenverantwortung als neoliberale Forderung dargestellt, welche die Soziale Arbeit herrschaftsstabilisierend domestiziert und im Namen eines schlanken Staates abbaut bzw. der Klientel Sozialer Arbeit Entsolidarisierung, grössere Unsicherheit und Selbstausbeutung zumutet. Vgl. auch Suitbert Cechura, Inklusion: Die Gleichbehandlung Ungleicher. Recht zur Teilhabe an der Konkurrenz, Münster 2015: Unter dem programmatischen Titel «Inklusion: Die Gleichbehandlung Ungleicher. Recht zur Teilhabe an der Konkurrenz» entfaltet er eine grundlegende Kritik an Inklusion als einer Gemeinschaftsideologie. Selbstbestimmung bedeute, gleichberechtigt teilhaben zu müssen an Arbeit und Geld bzw. am Konkurrenzkamp - bisheriger Schutz sozial Schwacher (insbesondere Behinderter) im Rahmen des Sozialstaats werde unter der Inklusionsideologie gemäss der Marktlogik umgebaut, was auf eine Markttotalisierung hinauslaufe.

18 Walter Schmid, Sozialarbeit und Eigenverantwortung: Das Empowerment-Konzept, in: Caritas Schweiz (Hg.), Sozialalmanach 2007. Schwerpunkt: Eigenverantwortung. Luzern 2006, 107-119.
Anstellungsträger» ${ }^{19}$ und Ressourcen sind ungleich verteilt. Zweitens spricht Schmid von der weiteren Gefahr, dass Soziale Arbeit sich mit Empowerment der eigenen Verantwortung entledigt - implizit blitzt hier auf, dass gelingendes Leben eben nicht nur aus individueller Selbstbestimmung besteht, sondern in einem zwischenmenschlichen, sozialen Raum von geteilter Verantwortung aufblüht.

Im gleichen Sozialalmanach schreibt der Psychologe Niklas Baer, Leiter der Fachstelle für psychiatrische Rehabilitation Baselland, zur Selbstbestimmung psychisch kranker Menschen. ${ }^{20}$ Einmal zeigt er anhand von Interviews mit Betroffenen, dass das «Erleben zwischen Autonomie und Abhängigkeit eine von mehreren Dimensionen darstellt. Der Wunsch nach Autonomie wird [...] nicht als prioritär beschrieben. ${ }^{21}$ Autonomie wird erlebt, wenn man würdig sowie respektvoll behandelt und ernst genommen wird - was nicht unbedingt rationale Selbstbestimmung oder gar äussere Selbständigkeit meint. «Die innere Autonomie ist ein Zeichen dafür, dass es gelungen ist, die Krankheit in das Selbstbild zu integrieren..) ${ }^{22}$ Dann arbeitet Baer sehr hellsichtig ein Paradox heraus: Wenn Gesunde definieren, dass für Kranke Selbstbestimmung höchste Priorität habe, nehmen sie sie gerade weg! Er geht noch weiter: Wenn Gesunde Eigenverantwortung von Kranken fordern und Krankheit zugunsten von Ressourcen bagatellisieren, dann entwürdigt dies die Betroffenen und lässt sie mit ihrem Leiden allein. ${ }^{23}$ Gesunde sollten vielmehr eigene Defi-

19 Avenir Suisse, Berufskodex (Anm. 12), 7

20 Niklas Baer, Gesundheit: Patient sein zwischen Abhängigkeit und Autonomie, in: Caritas Schweiz (Hg.), Sozialalmanach 2007. Schwerpunkt: Eigenverantwortung. Luzern 2006, 165-181.

21 Baer, Gesundheit (Anm. 20), 170

22 Baer, Gesundheit (Anm. 20), 174

23 Baer (Gesundheit [Anm. 20]) zeigt, dass psychisch Kranke diese Betonung grösserer Selbstverantwortung negativ erleben: Sie sind (mit) schuld an ihrer Krankheit. Er meint, dass Gesunde die Kranken stigmatisieren («selber schuld», «wollen nicht») - 
zite und Abhängigkeiten anerkennen; dann müssten sie sich nicht aus Selbstschutz von Kranken distanzieren, anstatt ihnen echt und nahe zu begegnen, wozu eben auch das anstrengende Aushalten der schwierigen Realität der Patienten und der Möglichkeit der eigenen Erkrankung gehört. Baer sagt zusammenfassend: «Oft ermöglicht Fürsorge eine stärkere Selbstbestimmung.» ${ }^{24}$ Wiederum steht hinter dieser ethischen Orientierung eine anthropologische Ellipse: Das selbstbestimmte Individuum in interdependenten Beziehungen von Geben und Nehmen.

Ähnlich argumentiert Dieter Röh, Professor für Sozialarbeitswissenschaft, zu Selbstbestimmung und Inklusion:

«Im Sinne der oben bereits angeführten Diskussion um Inklusionsbedingungen erscheint es sinnvoll, von einem ethischen Kontinuum auszugehen, welches auf der ei nen Seite Selbstbestimmung und Autonomie und auf der anderen Abhängigkeit und Bedürftigkeit beinhaltet. Dieses realistische Menschenbild ergänzt die Sicht auf das nach Autonomie und Selbstbestimmung strebende Individuum um seine vom sozialen Miteinander abhängige Bedürftigkeit. Menschen, ob mit oder ohne Behinderung, benötigen, um mit Ralf Dahrendorf zu sprechen, sowohl Optionen, um ihr Leben führen und gestalten zu können, als auch Ligaturen, durch die sie Halt, Unterstützung und Sicherheit erfahren. [...] Ethisches Handeln in der Behindertenhilfe is deshalb in das Kontinuum von Selbstbestimmung/Empowerment und Abhängigkeit/Verantwortung eingespannt, aus dem es keinen - höchstens einen idealisieren-

als Schutzmechanismus: «Bedürfnis [...] ein Fehlverhalten zu finden, das erklärt, warum diesem Menschen das passieren konnte (und uns nicht passieren kann).» (168) Die Betonung der Idee der Eigenverantwortung korreliere mit dem Bedürfnis, an ei ne gerechte Welt zu glauben (Menschen bekommen, was sie verdienen; guten Menschen geschehen gute Dinge und umgekehrt) bzw. dem Bedürfnis nach Sicherheit angesichts der Bedrohung durch Krankheit. «Die Idee gibt uns die scheinbare Sicherheit, dass uns nichts Schlimmes passieren kann, solange wir uns gut und richtig benehmen». (169)

24 Baer, Gesundheit (Anm. 20), 178. den oder hegemonialisierenden - Ausweg gibt. Idealisierend wäre dieser, wenn er menschliche Selbstbestimmung und Autonomie absolut setzte; hegemonialisierend, wenn Menschen mit paternalistischer Macht andere in abhängige Positionen zwängen.» ${ }^{25}$

\subsection{Biblisch-theologische Überlegungen}

Abschliessend folgen noch kurze Überlegungen zur Selbstbestimmung als ethischer Orientierung, als Orientierung für gelingendes Leben mit dem eigenen Selbst und dem Selbst anderer Menschen, wiederum strukturiert anhand der vier Akte des biblischen Dramas Schöpfung - Sündenfall - Erlösung - Vollendung. Selbstbestimmung angesichts des Selbst, das gewollt, gefährdet, geliebt ist und erwartet wird.

2.3.1 Schöpfung: Wir sind gewollt - Selbstbestimmung bejaht, ergänzt und zugespitzt

Gott hat das menschliche Selbst geschaffen. Zum gelingenden menschlichen Leben gehört darum die Selbst-bestimmung: Ein grosses Ja zu Individualität, Selb-ständigkeit und Selbst-entfaltung. Das neuzeitliche und aufklärerische Autonomiestreben und Individualismus, obwohl bisweilen anti-kirchlich zugespitzt und mit Widerstand von Teilen der Kirche, wächst ganz klar auch aus jüdisch-christlichen Wurzeln. Die Betonung der Selbstbestimmung in der Sozialen Arbeit, insbesondere die Förderung der Selbstbestimmung der Klientel innerhalb von Beziehungen mit einem Machtgefälle, hat ein klares biblisches Fundament.

Gott hat das menschliche Individuum als Beziehungswesen in Interdependenz mit anderen geschaffen. Darum wird die ethisch gebotene Selbstbestimmung ergänzt: Das Ja zur eigenen Begrenztheit und Ange-

25 Dieter Röh, Soziale Probleme, Selbstbestimmung und Inklusion, in: Sozialaktuell 5/Mai 2009, 13-16 (16) 
wiesensein auf andere führt erstens auch zu einem Ja zur Fremdbestimmung. ${ }^{26} \mathrm{Zu}$ einem gelingenden Leben gehört es, geliebt und umsorgt und anerkannt zu werden. Das Individuum lebt nicht nur aus dem eigenen Selbst, sondern empfängt auch von anderen. Eine so verstandene ethisch gebotene Fremdbestimmung rechtfertigt nicht etwa den Zwang als Antipode zu selbst-bestimmter Freiheit, sondern sie anerkennt den Wert der Verbundenheit als komplementär zu selbständiger Individualität. Dies gilt es in unserer gesamten Gesellschaft gegenüber der Gefahr eines übertriebenen Individualismus festzuhalten.

Dann hat Gott die Menschen mit einer Bestimmung geschaffen. Darum wird die ethisch gebotene Selbstbestimmung zweitens teleologisch zugespitzt, nämlich das menschliche Selbst soll Gott vertrauen und den Nächsten lieben (Mt 22,34-40, zitiert 5. Mose 6,5 und 3. Mose 19,18). Wahre Selbstbestimmung ist nicht egozentrisch oder blind für andere, sondern orientiert am Individuum-in-Beziehungen.

Die grundlegende anthropologische Polarität führt also zu einer Selbstbestimmung als ethischem Konzept, das in sich komplex bzw. spannungsvoll ist. Die anthropologische Ellipse soll, ethisch gesehen, das Individuum vor falschen Zwängen und Übergriffen anderer schützen; ebenso das soziale Wesen Mensch vor Egozentrik und falschem Individualismus; und sie soll verantwortliches Handeln des Individuum-inBeziehung fördern und fordern.

2.3.2 Sündenfall: Wir sind gefährdet - Selbstbestimmung kann sich selbst täuschen

Die ethisch gebotene Selbstbestimmung für ein gelingendes Leben ist gefährdet. Das menschliche Selbst kann sich über sich und andere täuschen.

26 Heine, Koordinaten (Anm. 11), spricht von begrenzter Autonomie, schon in der guten Schöpfung Gottes.
Selbstbestimmung kann sich in egoistische Selbstvergötzung verkehren, welche (1) Macht missbraucht, (2) andere abwertet, (3) Interdependenzen (Begrenzung und Bedürftigkeit) und (4) Solidarität (Beauftragung) verneint. Konkret: Sozialarbeitende können (1) übergriffig die asymmetrische Beziehung zur Klientel missbrauchen, (2) die Klientel negativ bemitleiden oder auf ihre Bedürftigkeit (oder sogar Defizite) festlegen und (3) die eigene Begrenzung ausblenden; die Klientel kann genauso (1) Macht missbrauchen, zum Beispiel durch Verweigerung, (2) die «andern» und das «Scheiss-system» verteufeln, (3) eigene Bedürftigkeit verleugnen und (4) sich der Verantwortung bzw. der Beziehungsorientierung entziehen.

Selbstbestimmung kann auch zur Forderung (gegenüber sich und/oder andern) der Selbsterlösung werden: Selbstverwirklichung und Selbstvervollkommnung sind das Gebot der Stunde - und wer das nicht schafft, ist eben selber schuld. ${ }^{27}$ Konkret: Sozialarbeitende können Klientel mit zu viel Selbstbestimmung überfordern, ihnen stützende Fremdbestimmung / Fürsorge vorenthalten, welche sie zur Selbstentfaltung benötigen, oder sich vor gemeinschaftlicher Mitverantwortung im Namen einer vermeintlichen Professionalität drücken.

Insofern akzentuiert die theologische Rede vom Sündenfall - «wir sind gefährdet» - das in der Schöpfung schon angelegte spannungsvolle, komplexe Konzept der Selbstbestimmung.

27 Diese Selbst-täuschung fokussiert den Menschen als Individuum auf Kosten de Menschen als Sozialwesen. Die umgekehrte Selbst-Täuschung ist auch möglich, führt aber über das Thema der Selbstbestimmung hinaus. Heine (Koordinaten [Anm. 11]) spricht auch im Zusammenhang des Sündenfalls von begrenzter Autonomie: Die sündenbedingte Gefährdung der Selbstbestimmung bleibt bestehen; Humanismus und Aufklärung gehen zu optimistisch von einem Menschen aus, der Sünde selber überwindet und darum unbegrenzt autonom sein will. 
2.3.3 Erlösung: Wir sind geliebt - Selbstbestimmung und Selbstverleugnung

Jesus Christus fordert von denjenigen, die ihm nachfolgen, die Selbstverleugnung: «Wenn einer mir auf meinem Weg folgen will, verleugne er sich und nehme sein Kreuz auf sich.» (Mt 16,24) Dies ist aber nicht der christliche Todesstoss gegen die Selbstbestimmung oder gar die christliche Rechtfertigung der Fremdbestimmung der Klientel (und sei sie sozialarbeiterisch noch so gut gemeint). Vielmehr meint Jesus, dass das selbstische Sein-Wollen-wie-Gott ans Kreuz gehört. Die Ursünde schlechthin soll hingerichtet werden, nämlich ein Selbst, das absieht von der Grundbeziehung zu Gott. Das Leben gelingt nicht, wenn das Selbst unabhängig und autark sein will, egoistisch um sich selber kreist, auf Kosten anderer lebt, ohne die Gott-gegebene ek-zentrische Zielrichtung. Dies alles soll das Selbst verleugnen und sich vielmehr anders, nämlich erlöst selbst bestimmen. Die Frucht des Heiligen Geistes ist, dass das Selbst sich selber beherrscht (Gal 5,11;2. Tim 1,7). Die Psalmisten führen Selbstgespräche mit ihrer Seele (z.B. Ps 42,5; 103,1) und nehmen so ihr eigenes Selbst an der Hand (Selbstführung!). Paulus mahnt die Ältesten in Ephesus, auf sich selbst zu achten (Apg 20,28) - und im Licht des NT bedeutet dies unter anderem, nicht falsch fremdbestimmt zu sein (Eph 4,14; Hebr 8,11) oder ungesund abhängig von anderen (Joh 5,44).

Also nochmals: In der Bibel und insbesondere auch im NT ist nicht die Selbstbeziehung an sich die Grundsünde. ${ }^{28}$ Vielmehr wird das menschliche Selbst, von Gott als Individuum-in-Beziehung geschaffen, hundertprozentig bejaht. Gleichzeitig wird das verbogene, zerbrochene Selbst nach dem Sündenfall hundertprozentig geliebt und nicht sich selbst, seiner Gefährdung und Selbstzerstörung überlassen. Weil das eigene Selbst und das Selbst der anderen von Gott geliebt sind, darum

28 Vgl. Huxel, Selbst (Anm. 10), 1155. wird die ethisch gebotene Selbstbestimmung durch die Erlösung in Jesus Christus davor befreit, in Selbstgerechtigkeit $(1$. Kor 4,4) oder Selbstverurteilung (1. Joh 3,19f; Röm 7,24 und 8,1) oder Verachtung bzw. Verurteilung anderer (Röm 14,10.15) abzugleiten. Christliche Selbstverleugnung als erlöste Selbstbestimmung (d.h. mit göttlich basiertem, realistischem und also wahren Selbstwert und Wertschätzung anderer) liebt Gott und den Nächsten.

Die Erlösung in Jesus Christus ist gemäss biblischem Zeugnis eine Einladung und ein Angebot für den Menschen: Zu glauben. Die Erlösung eröffnet einen Freiraum der Verantwortung. Dies impliziert unter anderem Glaubensfreiheit inklusive das Recht auf Irrtum und/oder Ablehnung; es impliziert ebenso, dass Glaube sowie entsprechendes Verhalten auf gar keinen Fall erzwungen werden darf. Konkret: Christliche Sozialarbeitende dürfen erstens auf keinen Fall im Namen Gottes (so wie sie ihn erkannt haben) der Klientel gegen deren eigenen Willen Werte überstülpen oder Verhalten mit Zwang vorschreiben, etwa im Umgang mit Sexualität oder Selbstschädigung oder Sterbehilfe. Sie sollen aber zweitens - genauso wie säkulare oder anders religiös geprägte Berufsleute - ihr Verständnis gelingenden Lebens in das Arbeitsbündnis mit der Klientel werbend einbringen (immer sorgfältig, eingedenk der asymmetrischen Beziehung). Sie müssen drittens ihrem Gewissen treu bleiben der Berufskodex spricht vom «dritten Mandat» ${ }^{29}$-, welches sie nicht nur im Spannungsfeld des ersten und zweiten Mandats steuert, nämlich der Vorgaben der Anstellungsträger und dem Begehren der Klientel, sondern auch zusätzliche Spannungsfelder eröffnen kann: Zwischen dem eigenen Gewissen und dem Arbeitgeber sowie zwischen dem eigenen Gewissen und der Klientel. Die Treue zum eigenen Gewissen, wozu christliche Sozialarbeitende durch die Schärfung des Gewissens an Gottes Wort

29 Avenir Social, Berufskodex (Anm. 12), 7. 
vielleicht besonders sensibilisiert sind, kann im Einzelfall dazu führen, dem Arbeitgeber ungehorsam zu sein (bitte aber deklariert und offen, nicht heimlich) oder eine bestimmte Anstellung zu verlassen. Es kann im Einzelfall auch heissen, Klientel an andere Berufsleute zu vermitteln oder die professionelle Tätigkeit transparent als «gegen die eigene Überzeugung, aber gemäss dem Willen der Klientel» zu deklarieren. Auf keinen Fall darf die Treue zum eigenen Gewissen die Selbstbestimmung des Arbeitgebers oder der Klientel aushebeln. Vielmehr leitet christliche Erlösung an, auch andere wirklich zu befreien und den Preis der damit verbundenen Möglichkeit der Ablehnung auf sich selber zu nehmen.

2.3.4 Vollendung: Wir werden erwartet - Selbstbestimmung im Licht des ewigen Selbst

Im Licht der Auferweckung von Jesus Christus an Ostern vor 2000 Jahren erwartet christliche Hoffnung die Auferstehung der Toten: Nicht nur einen neuen Himmel und eine neue Erde. Nicht nur die Vollendung des Reiches Gottes in Gerechtigkeit und Frieden. Sondern auch die vollkommene Erlösung des Individuums als Leib und Seele:30 Die leibliche Auferstehung des individuellen Leibes (1. Kor 15,12ff) und die Verleihung eines neuen, persönlichen und individuellen Namens (Offb 2,17), in Fortsetzung des eigenen Namens, der im Buch des Lebens eingetragen ist (Lk 10,20; Offb 20,15; Dan 12,1). So wertvoll ist Gott das menschliche Selbst, dass er es in Herrlichkeit vollendet und eine ewige Zukunft bereitet. Auf dieses Ziel hin erfolgt die ethisch gebotene Selbstbestimmung schon heute und verleiht dem unendlichen Wert des Individuums eine ungeahnte Dimension.

30 Vgl. Dietrich Bonhoeffers Gedicht «Stationen auf dem Weg zur Freiheit»: «Komm nun, höchstes Fest auf dem Wege zur ewigen Freiheit, / Tod, leg nieder beschwerliche Ketten und Mauern / unsres vergänglichen Leibes und unsrer verblendeten Seele, / dass wir endlich erblicken, was hier uns zu sehen missgönnt ist.»
Dies impliziert konkret für christliche Sozialarbeitende: Hoffnungsvolle Geduld bzw. geduldige Hoffnung! Sowohl sich selbst gegenüber als auch gegenüber der Klientel bleibt Selbstbestimmung komplex. Jeder Mensch als Individuum-in-Beziehung trägt diese spannungsvolle Polarität in sich: unendlich wertvoll - begrenzt - bedürftig - beauftragt. Die Ellipse verformt sich immer wieder zum Kreis, die potentielle Gefährdung wird immer wieder zur realen Selbst-Beeinträchtigung und -Zerstörung. Aber die Erlösung ist wirklich angebrochen: Die Kraft Gottes als gegenwärtiger Heiliger Geist ermöglicht Unmögliches, vergibt und heilt Vergangenes, eröffnet zweite und dritte und x-te Chancen. Das gibt Hoffnung für verfahrene Situationen, für sogenannt hoffnungslose Fälle, für die eigenen Fettnäpfchen mit ihrer magnetischen Anziehungskraft. Die Erlösung wird einmal vollendet und lässt darum heute schon geduldig Begrenzungen mit-tragen, Nicht-Wollen bzw. Nicht-Können aushalten, den für menschliche Augen bisweilen zugeschütteten Wert jedes Menschen immer wieder ausgraben und ausdauernd an die Teilhabe jedes Menschen an der grossen Bestimmung des Selbst als Individuum-in-Beziehung festzuhalten.

Zusammenfassend: Vielleicht sind die vier Akte des biblischen Dramas zur Selbstbestimmung als komplexen ethischen Konzept für christliche Sozialarbeitende ein wenig wie Ovo: «Mit Ovo kannst Du es nicht besser, aber länger». Schöpfung und Sündenfall, «du bist gewollt» und «du bist gefährdet», vertiefen und konturieren die auch sozialwissenschaftlich bekannte Polarität des Menschen als Individuum und soziales Wesen. Erlösung und Vollendung, «du bist geliebt» und «du wirst erwartet» geben eine weitere Dimension von Kraft und Hoffnung auf dem professionellen Weg, inmitten aller Spannungen die Klientel bei der Verwirklichung ihres Selbst als Individuum-in-Beziehung zu unterstützen und die eigene Selbstbestimmung zu leben.

Ganz zum Schluss noch ein poetischer Text von Hans-Joachim Eckstein, Theologieprofessor in Tübingen: 
«Wer sich in seinem Glauben von Gott her versteht, der wird sich auch selbst verständlich. Wer Gott selbstvergessen von Herzen lieben will, der wird gerade dadurch selbstbewusst. Und wer in Gott seine Mitte hat, ist ganz bei sich selbst. Das heisst nicht etwa, dass wir selbst göttlich wären, sondern schlicht, dass wir als Gottes Gegenüber, d.h. als seine Ebenbilder, Gott endlich Gott sein lassen und uns seine Menschen.» ${ }^{31}$

Autor:

Paul Kleiner,

Dr. theol., Pfarrer in der reformierten Kirchgemeinde Pfäffikon (ZH), ehem.

Rektor des Theologisch-Diakonischen Seminars (TDS) Aarau

31 Hans-Joachim Eckstein, Du bist ein Wunsch, den Gott sich selbst erfüllt hat, Holzgerlingen $42016,91$. 